\title{
MOLECULAR DYNAMICS SIMULATIONS OF NANOSTRUCTURED CERAMIC MATERIALS ON PARALLEL COMPUTERS
}

\author{
Final Progress Report \\ (March 1996 - August 2003)
}

Priya Vashishta and Rajiv K. Kalia

Concurrent Computing Laboratory for Materials Simulations

Department of Physics and Astronomy

Department of Computer Science

Louisiana State University, Baton Rouge, LA 70803-4001

Phone: (225) 578-1342; Fax: (225) 578-5855

E-mail: (priyav, kalia)@bit.csc.lsu.edu

PREPARED FOR THE U.S. DEPARTMENT OF ENERGY

UNDER GRANT NUMBER DE-FG02-96ER 45570 


\title{
PROGRESS REPORT (March 1996 - August 2003)
}

\author{
PROPOSAL TITLE: Molecular Dynamics Simulations of Nanostructured Ceramic \\ Materials on Parallel Computers \\ GRANT NUMBER: DE-FG02-96ER 45570 \\ PRINCIPAL \\ INVESTIGATORS: Priya Vashishta and Rajiv K. Kalia \\ INSTITUTION: $\quad$ Concurrent Computing Laboratory for Materials Simulations \\ Department of Physics and Astronomy \\ Department of Computer Science \\ Louisiana State University, Baton Rouge, LA 70803-4001 \\ Phone: (225) 578-1342; Fax: (225) 578-5855 \\ E-mail: (priyav, kalia)@bit.csc.lsu.edu
}

\section{EXECUTIVE SUMMARY}

Large-scale molecular-dynamics (MD) simulations have been performed to gain insight into: i) sintering, structure, and mechanical behavior of nanophase $\mathrm{SiC}$ and $\mathrm{SiO}_{2}$; ii) effects of dynamic charge transfers on the sintering of nanophase $\mathrm{TiO}_{2}$; iii) high-pressure structural transformation in bulk $\mathrm{SiC}$ and GaAs nanocrystals; iv) nanoindentation in $\mathrm{Si}_{3} \mathrm{~N}_{4}$; and v) lattice mismatched InAs/GaAs nanomesas. In addition, we have designed a multiscale simulation approach that seamlessly embeds MD and quantum-mechanical (QM) simulations in a continuum simulation.

The above research activities have involved strong interactions with researchers at various universities, government laboratories, and industries. 33 papers have been published and 22 talks have been given based on the work described in this report. 


\section{§1 RESEARCH ACCOMPLISHMENTS}

In the past years we have performed large-scale molecular-dynamics (MD) simulations to investigate: i) sintering, structure, and mechanical behavior of nanophase $\mathrm{SiC}$ and $\mathrm{SiO}_{2}$; ii) effects of dynamic charge transfers on the sintering of nanophase $\mathrm{TiO}_{2}$; iii) high-pressure structural transformation in bulk $\mathrm{SiC}$ and GaAs nanocrystals; iv) nanoindentation in $\mathrm{Si}_{3} \mathrm{~N}_{4}$; and v) lattice mismatched InAs/GaAs nanomesas. In addition, we have designed a multiscale simulation approach that seamlessly embeds MD and quantum-mechanical (QM) simulations in a continuum simulation. These accomplishments are briefly described in this section.

\section{\$1.1 STRUCTURE AND MECHANICAL PROPERTIES OF NANOPHASE CERAMICS}

Advanced structural ceramics are highly desirable materials for applications requiring extreme operating conditions. Light-weight, elevated melting temperatures, high strengths, and wear and corrosion resistance make them very attractive for high-temperature and high-stress applications. The only serious drawback of ceramics is that they are brittle at low to moderately high temperatures.

In recent years a great deal of progress has been made in the synthesis of ceramics that are much more ductile than conventional coarse-grained materials. These so called nanophase materials are fabricated by in-situ consolidation of nanometer size clusters. Despite a great deal of research, many perplexing questions concerning nanophase ceramics remain unanswered. Experiments have yet to provide information regarding the morphology of pores or the structure and dynamics of atoms in nanophase ceramics. As far as modeling is concerned, only a few atomistic simulations of nanophase materials have been reported thus far. This is due to the fact that these simulations are computationally very demanding: as realistic simulation of a nanophase solid requires $10^{5}-10^{6}$ time steps for processing and $\sim 10^{6}$ atoms since each nanocluster itself consists of $10^{3}-10^{4}$ atoms.

Molecular dynamics simulations have been performed to investigate the structure and mechanical behavior of nanophase $\mathrm{SiC}$ and $\mathrm{SiO}_{2}$. Interatomic potentials in these simulations consist of two- and three-body terms. The two-body terms are steric repulsion and screened Coulomb potentials due to charge transfer between atoms, and a charge-dipole interaction that takes into account the large electronic polarizability of anions. The three-body terms take into account covalent effects through bond-bending and bond-stretching terms. These interatomic interactions are validated by comparing the MD results with a variety of experimental measurements. We find: i) bond lengths and bond-angle distributions in crystalline systems are in excellent agreement with experiments; ii) positions and relative heights of various peaks in the static structure factor for amorphous $\mathrm{SiC}$ and $\mathrm{SiO}_{2}$ are in good agreement with neutron scattering measurements; iii) phonon densities-of-states of crystalline and amorphous systems agree well with inelastic neutron scattering experiments; and iv) elastic moduli of crystalline and amorphous systems are also in good agreement with experimental values.

Recently we performed the first joint experimental and MD study of nanophase SiC (n$\mathrm{SiC}$ ). Both experiment (solid diamonds in Fig. 1) and simulations (open circles in Fig. 1) reveal the onset of sintering around $1500 \mathrm{~K}$. The MD simulations provide a microscopic picture of how 
the morphology of micropores in $\mathrm{n}-\mathrm{SiC}$ changes with pressure. The fractal dimension and the surface roughness exponent of micropores are found to be 2.4 and 0.45 , respectively, over the entire pressure range between 0 and $15 \mathrm{GPa}$. Small-angle neutron scattering at low wavevectors yields a fractal dimension of 2 for pores in $\mathrm{n}-\mathrm{SiC}$. MD calculations of pair-correlation functions and bond-angle distributions reveal that interfacial regions between nanoparticles are highly disordered with nearly the same number of 3-fold and 4-fold coordinated Si atoms. Atomic diffusivities of $\mathrm{Si}$ and $\mathrm{C}$ in inter-particle regions are considerably larger than those inside the particles. The effect of consolidation on mechanical properties is also investigated with the MD approach. The results show a power-law dependence of elastic moduli on the density with an exponent of $3.4 \pm 0.1$.

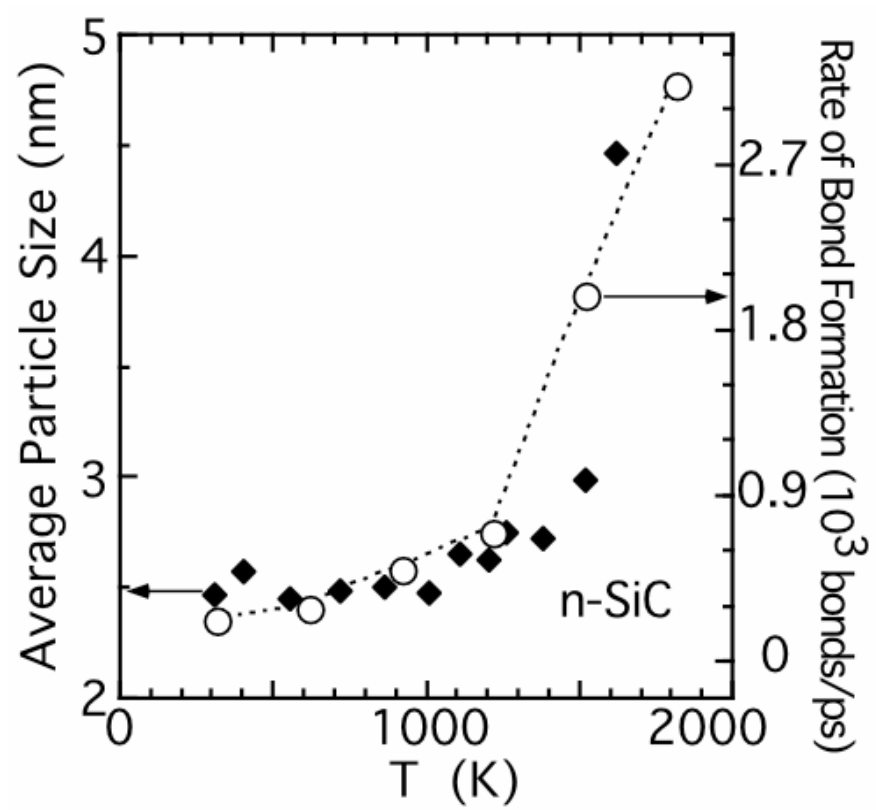

Fig. 1: The onset of sintering is indicated by an increase in the average particle size in the neutron data $(\checkmark)$ and an increase in the rate of bond formation between nanoparticles in the MD results (o). The dotted line is a guide to the eye for the MD results.

The simulation of nanophase $\mathrm{SiO}_{2}$ involves amorphous nanoclusters which are obtained from bulk amorphous $\mathrm{SiO}_{2}$. By sintering at different pressures, we generated nanophase solids with densities ranging from $76 \%$ to $93 \%$ of the bulk amorphous density $\left(2.2 \mathrm{~g} / \mathrm{cm}^{3}\right)$. In these solids the morphology of micropores, mechanical behavior, and the effect of nanoscale structures on the short-range and intermediate-range order (SRO and IRO) are investigated. Pores in nanophase $\mathrm{a}-\mathrm{SiO}_{2}$ are found to have a self-similar structure with a fractal dimension close to 2 and the pore surface width scales with the volume as, $W \sim \sqrt{ } V$. The MD simulations reveal that the SRO in nanophase silica glass is very similar to that in the bulk glass: both of them consist of corner-sharing $\mathrm{Si}\left(\mathrm{O}_{1 / 2}\right)_{4}$ tetrahedra. However, the IRO in nanophase silica glass is quite different from that in the bulk glass. In the nanophase silica glasses the first sharp diffraction peak (FSDP), the signature of IRO, has a much smaller height and is shifted to smaller wavevectors relative to the FSDP in the bulk silica glass. From the partial static structure factors and pair distribution functions, we find that $\mathrm{Si}-\mathrm{O}$ and $\mathrm{Si}-\mathrm{Si}$ correlations in the range of $4-10 \AA$ are primarily responsible for differences in the IRO of bulk and nanophase silica glasses. We have 
also investigated the mechanical behavior of nanophase a- $\mathrm{SiO}_{2}$. The elastic moduli are found to have a power law dependence on the density with an exponent of 3.5. These results are in excellent agreement with experimental measurements on high-density silica aerogels.

\section{\$1.2 DYNAMic Charge Transfer AND SinTERING IN $\mathrm{TiO}_{2} \mathrm{NANOPARTICLES}^{-2}$}

We have also determine structural and thermo-mechanical properties of nanostructured titania $\left(\mathrm{n}-\mathrm{TiO}_{2}\right) . \quad \mathrm{n}-\mathrm{TiO}_{2}$ has technological applications in solar cells, cancer therapy, and in selfsterilizing surgery rooms. $\mathrm{n}-\mathrm{TiO}_{2}$, a promising photovoltaic material, requires a variable-charge interaction scheme because of significant charge fluctuations in interfacial regions. Recently we have developed such an approach and validated it by comparing the MD calculations with experiments. Lattice constant, cohesive energy, and elastic moduli are in good agreement with experimental results. The interaction potential also describes the dielectric properties of rutile $\mathrm{TiO}_{2}$ correctly. The MD results for dielectric constants, $\varepsilon_{x x}=92$ and $\varepsilon_{z z}=196$, of the rutile crystal agree well with experimental values $\left(\varepsilon_{x x}=86\right.$ and $\left.\varepsilon_{z z}=170\right)$ and the directionallyaveraged dielectric constant of the anatase crystal $(\varepsilon=46)$ also agrees well with the experimental value $(\varepsilon=48)$. We have investigated surface-structure stabilities and space-charge distributions of both rutile and anatase phase $\mathrm{TiO}_{2}$ nanoparticles. MD simulations reveal that dynamic charge transfer enhances high-temperature atomic diffusion on surfaces of $\mathrm{TiO}_{2}$ nanoparticles and creates an additional repulsive force due to a double-charge surface layer between nanoparticles.

Variable-charge MD simulations have also been performed to study sintering of $\mathrm{TiO}_{2}$ nanoparticles. We found that the sintering of two anatase-nanospheres is influenced predominantly by enhanced surface diffusion at high temperatures.
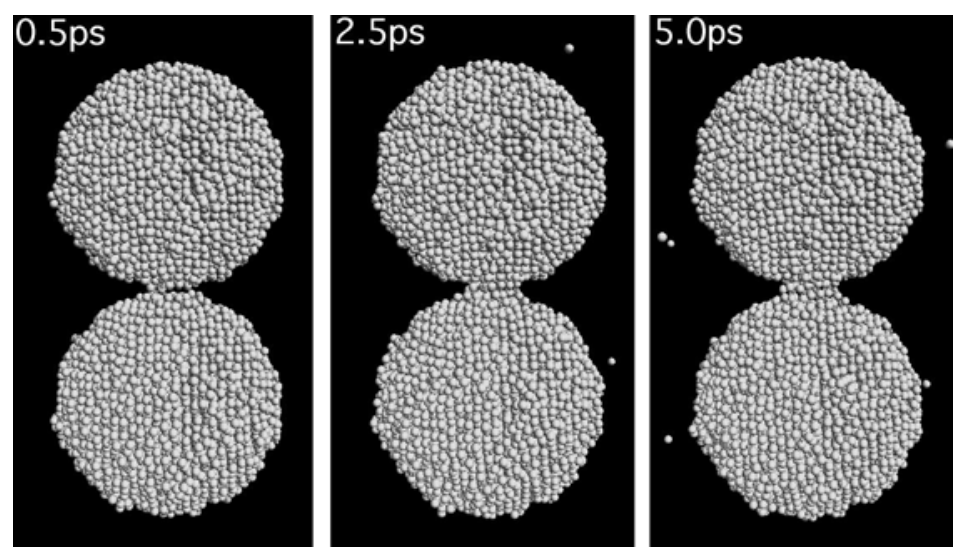

Fig. 2: Atomic configurations of two anatase nanospheres $(d=60 \AA)$ in the MD simulations at $1,400 \mathrm{~K}$ with the $<100>-<100>$ orientation. Only $\mathrm{O}$ atoms are shown by spheres.

\section{§1.3 PRESSURE-INDUCED STRUCTURAL TRANSFORMATIONS}

Despite numerous experimental and theoretical studies, structural transformations in GaAs and $\mathrm{SiC}$ at high pressures are not well understood at the atomistic level. We have investigated the mechanisms of these transformations using an isothermal-isobaric MD approach and the interatomic potential schemes mentioned above. In $\mathrm{SiC}$, a reversible transformation between the 
four-fold coordinated zinc-blende structure and the six-fold coordinated rocksalt structure is found at a pressure of $100 \mathrm{GPa}$. The calculated volume change at the transition and the hysteresis are in good agreement with experimental data. The atomistic mechanism for the structural transformation is a cubic-to-monoclinic unit-cell transformation and a relative shift of $\mathrm{Si}$ and $\mathrm{C}$ sublattices in the [100] direction. The new transition path does not involve any bond breaking and it has a significantly lower activation energy compared with a previously proposed transformation mechanism (Fig. 3).

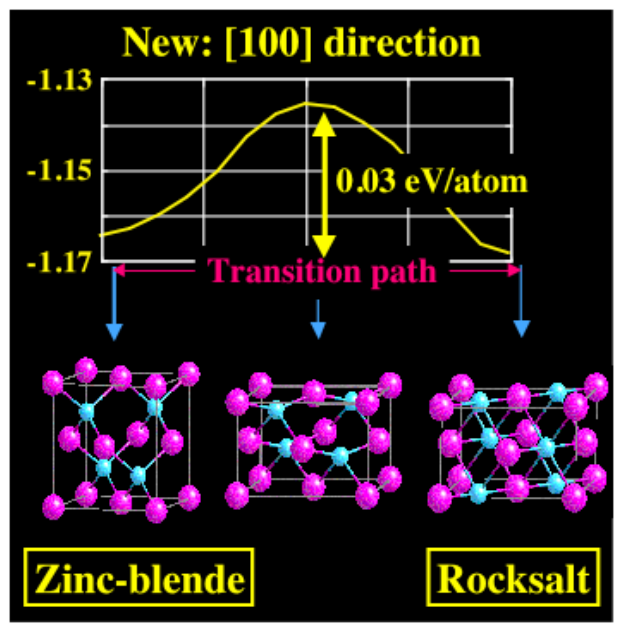

Fig. 3: The newly proposed transition path for the pressure-induced zinc-blende-to-rocksalt transformation in $\mathrm{SiC}$ (right) involves a relative shift of $\mathrm{Si}$ and $\mathrm{C}$ sublattices in the [100] direction. It has a small activation enthalpy $(0.03 \mathrm{eV} /$ atom $)$.

In crystalline GaAs, the pair-distribution functions and bond-angle distributions indicate that the four-fold coordinated zinc-blende structure transforms into a six-fold coordinated orthorhombic structure at a pressure of $22 \mathrm{GPa}$. The reverse transformation from the orthorhombic to zinc-blende structure shows hysteresis and is observed at a pressure of $\sim 10$ GPa. These MD results are in good agreement with experiments (Fig. 4).

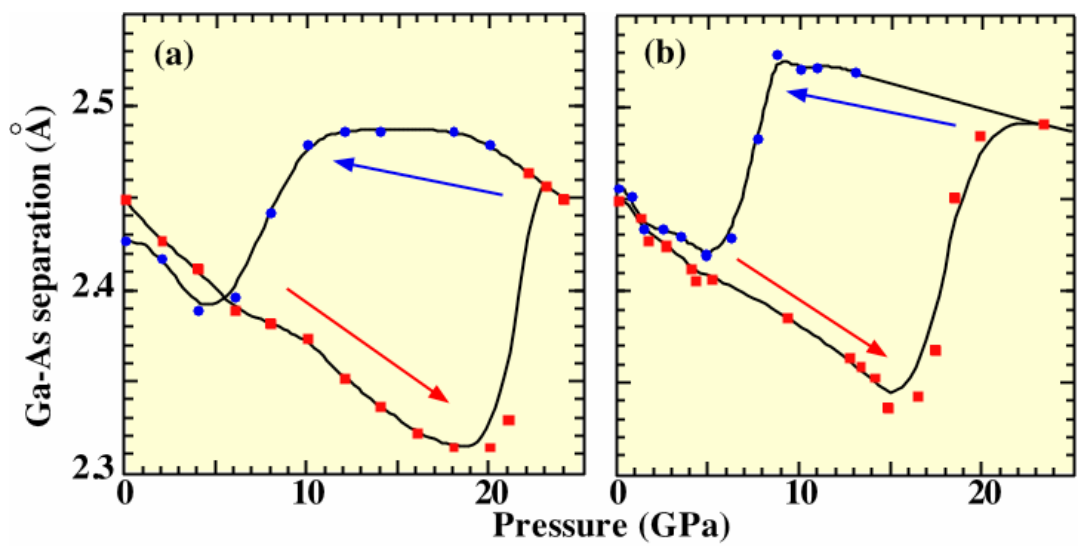

Fig. 4: Comparison between (a) MD and (b) EXAFS experimental results for the Ga-As nearestneighbor distance during forward (squares) and reverse (circles) structural transformations. 
We have also investigated pressure-induced structural transformations in GaAs nanocrystals of different sizes using parallel MD simulations. It is found that the transformation from four-fold (zinc blende) to six-fold (rocksalt) coordination starts at the surfaces of nanocrystals and proceeds inwards with increasing pressure. Inequivalent nucleation of the rocksalt phase at different sites leads to an inhomogeneous deformation of the nanocrystal. For sufficiently large spherical nanocrystals, this gives rise to rocksalt structures of different orientations separated by grain boundaries (see Fig. 5). The absence of such grain boundaries in a faceted nanocrystal of moderate size indicates sensitivity of the transformation to the initial nanocrystal shape. The pressure corresponding to the complete transformation increases with the nanocrystal radius and it approaches the bulk value for a spherical nanocrystal of $\sim 5,000$ atoms.

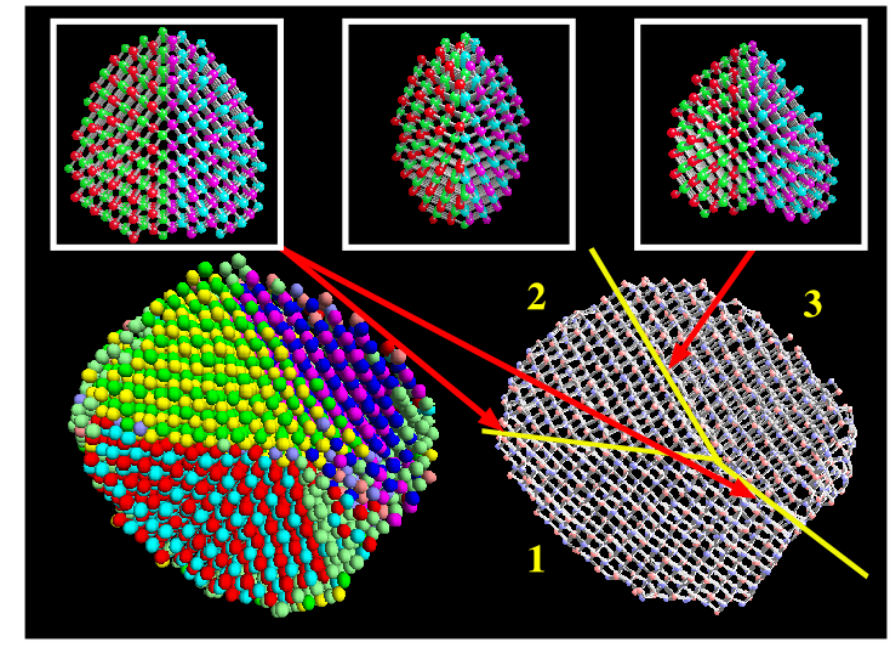

Fig. 5: Bottom left panel shows color-coded grain structures in a GaAs nanoparticle at a pressure of $22.5 \mathrm{GPa}$. Bottom right panel and insets show different grain boundary types.

\section{§1.4 NANOINDENTATION IN $\mathrm{Si}_{3} \mathrm{~N}_{4}$}

Nanoindentation testing is a unique local probe of mechanical properties of materials at surfaces and multilayered structures. This technique is especially useful in testing surfaces and thin films in microelectronics industry. For example, it is used for measuring critical stresses for dislocation generation in semiconductor devices. The importance of atomic-level understanding of indentation processes is widely recognized.

Using MD simulations, we are investigating nanoindentation in $\mathrm{Si}_{3} \mathrm{~N}_{4}$. The nanoindentation simulation is performed on the (0001) surface of a $60 \mathrm{~nm} \times 60 \mathrm{~nm} \times 30 \mathrm{~nm}$ crystalline $\tilde{\alpha} \mathrm{Si}_{3} \mathrm{~N}_{4}$ slab consisting of 10 million atoms (Fig. 6). The sample is indented using a pyramid indenter with a load $\sim 10 \mathrm{~N}$ and indentation depth $\sim 10 \mathrm{~nm}$. From the loaddisplacement curve, hardness value is estimated to be of $50.3 \mathrm{GPa}$. (We have also calculated the hardness of amorphous $\mathrm{Si}_{3} \mathrm{~N}_{4}$ to be $31.5 \mathrm{GPa}$ using a similar geometry.) Our simulations reveal significant plastic deformation and pressure-induced amorphization under the indenter. The simulations also exhibit anisotropic fracture toughness: Indentation cracks are observed along 
the $[\overline{1} 2 \overline{1} 0]$ direction, which coincides with one of the diagonal directions of the indenter, but not for the other diagonal direction, [1 $\overline{1} 00]$.

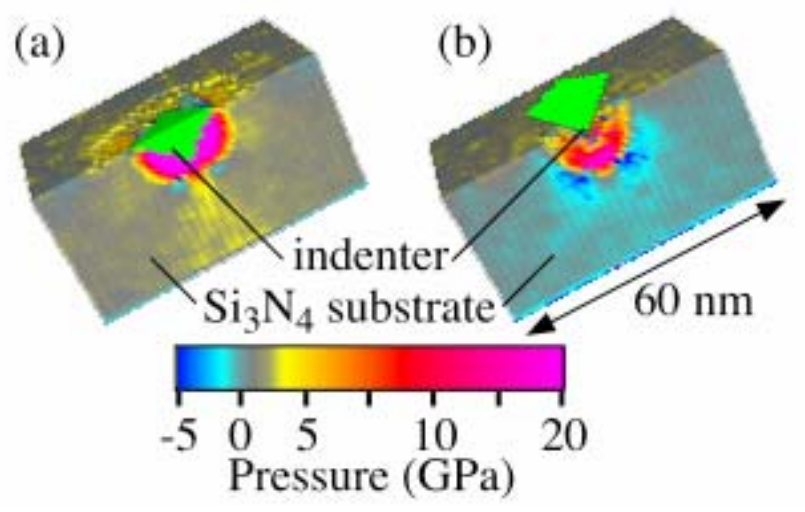

Fig. 6: Pressure distribution in $\mathrm{Si}_{3} \mathrm{~N}_{4}$ during (a) loading and (b) unloading of a nanoindenter.

\section{$\$ 1.5$ InAs/GaAs NANOMESAS}

We have performed multimillion-atom MD study of epitaxial InAs quantum dots on GaAs substrate. On infinite planar GaAs (001) substrates, deposition of the 7\% lattice mismatched InAs leads to the formation of coherent three-dimensional island structures above a critical amount, $\sim 1.6$ monolayers (ML), in order to partially relieve the strain. By contrast, previous experiments by Madhukar et al. at the University of Southern California have shown that, when InAs is deposited on $<100>$ oriented GaAs square mesas of size $\leq 75 \mathrm{~nm}$, due to significant strain accommodation in both lateral dimensions in the nanoscale mesa, the island morphology is suppressed, leading to a continuous film with flat morphology.

This InAs film growth is, however, self-limiting and stops at $\sim 11 \mathrm{ML}$. In order to understand the self-limiting nature of the InAs film growth, we have performed MD simulations of InAs/GaAs nanomesas with $\{101\}$-type sidewalls, see Fig. 7. We find that the in-plane lattice constant of InAs layers parallel to the InAs/GaAs(001) interface starts to exceed the InAs bulk value at the $12^{\text {th }} \mathrm{ML}$ and the hydrostatic stresses in InAs layers become tensile above $\sim 12^{\text {th }}$ ML. As a result, it is not favorable to have InAs overlayers thicker than $12 \mathrm{ML}$. This may explain the experimental findings of the growth of flat InAs overlayers with self-limiting thickness of $11 \pm 1 \mathrm{ML}$ on GaAs nanomesas. 


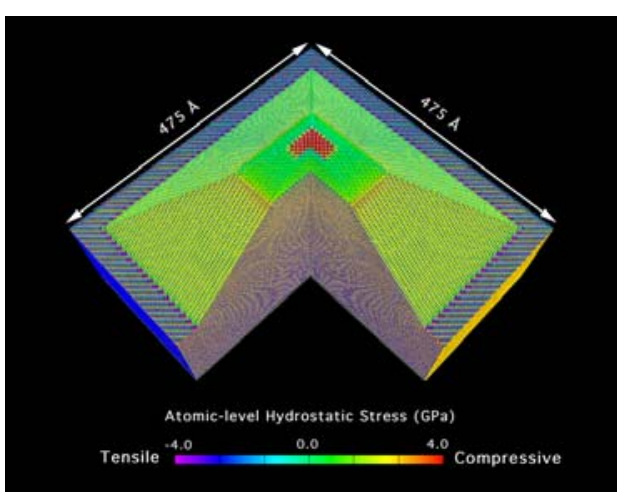

Fig. 7: Atomic-level hydrostatic stress in an InAs/GaAs square nanomesa with a 12 ML InAs overlayer.

Length scales are of critical significance for stress relaxation and manipulation leading to control of the island number on chosen nanoscale area arrays. For example, experiments at USC have shown that, on stripe mesas of sub-100-nm widths on GaAs(001) substrates, deposition of InAs can be made to result in the self-assembly of three, two, and single chains of InAs 3D island quantum dots selectively on the stripe mesa tops only for widths decreasing from $100 \mathrm{~nm}$ down to $30 \mathrm{~nm}$.

We have recently investigated lateral size effects on the stress distribution and morphology of InAs/GaAs nanomesas using parallel MD simulations. Two mesas with the same vertical size but different lateral sizes are simulated. For the smaller mesa, a single stress domain is observed in the InAs overlayer, whereas two stress domains are found in the larger mesa (a highly compressive domain is located at the center of the InAs overlayer, whereas the peripheral region of the InAs overlayer is less compressive). This indicates the existence of a critical lateral size for domain formation in accordance with recent experimental findings. We have also studied the morphology of the InAs overlayer near the InAs/GaAs interface. For a 2.2 million-atom nanomesa, the As layer is "dome" shaped. In contrast, the As layer in a 8.5 million-atom nanomesa shows a "dimple" at the center of the mesa; see Fig. 7. This provides clear evidence that there exists a critical lateral size for such stress domain formation and the critical value is somewhere between $124 \AA$ and $407 \AA$.

\section{§1.6 Multiscale ATomistic/ContinUUm Simulation AlgorithMS}

We have developed a multiscale simulation approach to study materials processes involving a hierarchy of length scales. This approach incorporates electronic processes over length scales of a few nm, atomistic processes over submicron dimensions, and continuum processes beyond the submicron length scale. The approach involves melding of a $10^{2}-10^{4}$-atom quantum mechanical (QM) calculation based on the linear-scaling density functional theory (DFT), a $10^{8}-10^{9}$ atom MD simulation, and continuum models described by the finite element (FE) method.

In the multiscale DFT/MD scheme, a QM region with $10^{2}-10^{4}$ atoms is embedded in a submicron size atomistic region. The DFT calculations are performed in real space using a highorder finite difference method with multigrid acceleration and a high-resolution grid adaptively 
generated near the atoms. This approach is embedded in MD simulations. The total potential energy $E_{\text {pot }}$ of the combined QM/MD system is obtained from,

$$
E_{\mathrm{pot}}=E_{\mathrm{CL}}^{\mathrm{system}}+E_{\mathrm{QM}}^{\text {cluster }}-E_{\mathrm{CL}}^{\text {cluste }}
$$

where $E_{\mathrm{CL}}^{\text {system }}$ is the classical MD potential energy for the whole system and the last two terms encompass the $\mathrm{QM}$ correction to that energy. $E_{\mathrm{QM}}^{\text {cluster }}$ and $E_{\mathrm{CL}}^{\text {cluster }}$ are the $\mathrm{QM}$ and classical MD energies of an atomic cluster cut out of the total system, respectively.

We have also developed a hybrid $\mathrm{MD} / \mathrm{FE}$ scheme to describe multiscale phenomena spanning atomistic to macroscopic length scales. Within the MD region, the dynamics of atoms are governed by suitable interatomic potentials and in the FE region the equations for continuum elastic dynamics are solved on a computational grid. The overlapping handshake (HS) region between MD and FE is suitably adjusted to serve as the inter-connector through which information flows both ways. Specifically, the FE grid in the HS region is refined down to the atomic scale with each FE node having a one-to-one correspondence with an MD atom. To ensure smooth transition, the dynamics of hybrid atoms/nodes are derived from suitably averaged MD and FE Hamiltonians.

The hybrid MD/FE simulation approach has been used to study stress distributions in silicon/silicon-nitride nanopixels, see Fig. 8. The hybrid approach provides atomistic description near the interface and continuum description deep into the substrate, increasing the accessible length scales and greatly reducing the computational cost. The results of the hybrid simulation are in good agreement with full multimillion-atom MD simulations: atomic structures at the lattice-mismatched interface between amorphous silicon nitride and silicon induce inhomogeneous stress patterns in the substrate that cannot be reproduced by a continuum approach alone

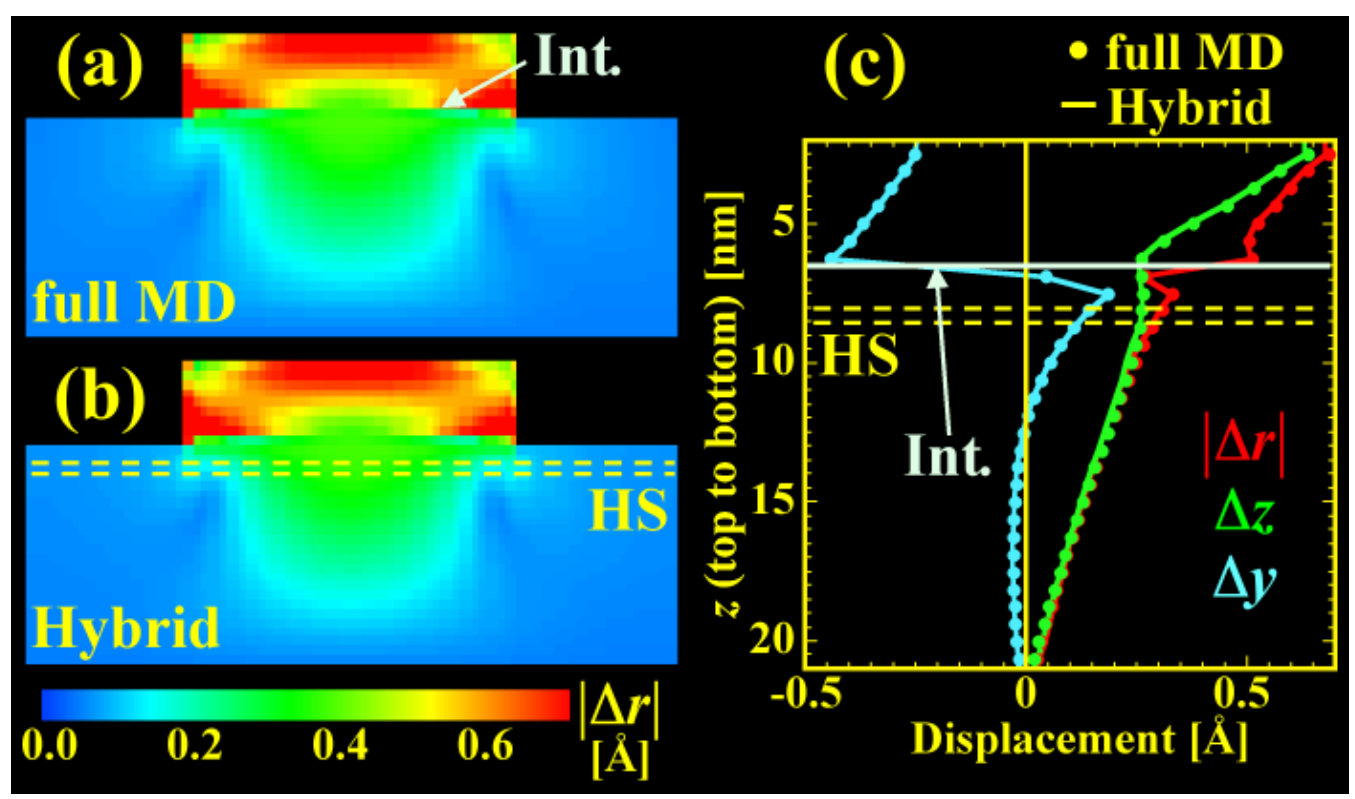


Fig. 8: Absolute displacement from equilibrium $|\Delta r|$ (averaged over a cell) color-coded on a slice through the center of the pixel defined by the Si [111] (also denoted as $z$ ) and [0-11] (also denoted as $y$ ) directions, for (a) the full MD, and, (b) the hybrid schemes. In (c), the displacements as a function of the $z$ coordinate (at $x=0, y=d / 4$, where $d=25 \mathrm{~nm}$ is the pixel width and $x=y=0$ is its center) for both systems are plotted. The $\mathrm{Si} / \mathrm{Si}_{3} \mathrm{~N}_{4}$ interface is marked by the horizontal gray line. The HS region for the hybrid system is $10 \AA$ below the top of the Si substrate.

\section{§2 PUBLICATIONS}

\section{BOOK}

- $\quad$ Computer-Aided Design of High-Temperature Materials (Oxford University Press, Oxford, 1999), Editors: A. Pechenik, R. K. Kalia, and P. Vashishta, and R. K. Kalia. 


\section{ARTICLES}

1. "Structural correlations and mechanical behavior in nanophase silica glasses," T. Campbell, R. K. Kalia, A. Nakano, F. Shimojo, K. Tsuruta, and P. Vashishta, Physical Review Letters 82, 4018 (1999).

2. "Dynamics of oxidation of aluminum nanoclusters using variable charge moleculardynamics simulations on parallel computers," T. Campbell, R. K. Kalia, A. Nakano, P. Vashishta, S. Ogata, and S. Rodgers, Physical Review Letters 82, 4866 (1999).

3. "Variable-charge interatomic potentials for molecular-dynamics simulations of $\mathrm{TiO}_{2}$," $\mathrm{S}$. Ogata, H. Iyetomi, K. Tsuruta, F. Shimojo, R. K. Kalia, A. Nakano, and P. Vashishta, Journal of Applied Physics 86, 3036 (1999).

4. "Dynamic fracture analysis," P. Vashishta and A. Nakano, Computing in Science \& Engineering 1 (5), 20 (1999).

5. "Scalable molecular-dynamics, visualization, and data-management algorithms for materials simulations," A. Nakano, R. K. Kalia, and P. Vashishta, Computing in Science \& Engineering 1 (5), 39 (1999).

6. "Large-scale atomistic simulation of dynamic fracture," P. Vashishta, R. K. Kalia, and A. Nakano, Computing in Science \& Engineering 1 (5), 56 (1999).

7. "Pressure induced structural transformation in nanocluster assembled gallium arsenide," S. Kodiyalam, A. Chatterjee, I. Ebbsjö, R. K. Kalia, H. Kikuchi, A. Nakano, J. P. Rino, and P. Vashishta, Materials Research Society Symposium Proceedings 536, 545 (1999).

8. "Molecular dynamics simulations of nanoindentation of silicon nitride," P. Walsh, A. Omeltchenko, H. Kikuchi, R. K. Kalia, A. Nakano, and P. Vashishta, Materials Research Society Symposium Proceedings 539, 119 (1999).

9. "Stress domains in $\mathrm{Si}(111) / \mathrm{Si}_{3} \mathrm{~N}_{4}(0001)$ nanopixel - 10 million-atom molecular dynamics simulations on parallel computers," A. Omeltchenko, M. E. Bachlechner, A. Nakano, R. K. Kalia, P. Vashishta, I. Ebbsjö, A. Madhukar, and P. Messina, Physical Review Letters 84, 318 (2000).

10. "Dislocation emission at silicon/silicon nitride interface - a million atom molecular dynamics simulation on parallel computers," M. E. Bachlechner, A. Omeltchenko, A. Nakano, R. K. Kalia, P. Vashishta, I. Ebbsjö, A. Madhukar, Physical Review Letters 84, 322 (2000).

11. "Molecular dynamics simulation of pressure induced structural transformation in silicon carbide,” F. Shimojo, I. Ebbsjö, R. K. Kalia, A. Nakano, J. P. Rino, and P. Vashishta, Physical Review Letters 84, 3338 (2000). 
12. "Sintering, structure and mechanical properties of nanophase $\mathrm{SiC}$ : a molecular-dynamics and neutron scattering study," A. Chatterjee, R. K. Kalia, C.-K. Loong, A. Nakano, A. Omeltchenko, K. Tsuruta, P. Vashishta, M. Winterer, and S. Klein, Applied Physics Letters 77, 1132 (2000).

13. "Amorphization and anisotropic fracture dynamics during nanoindentation of silicon nitride - a multi-million atom molecular dynamics study," P. Walsh, R. K. Kalia, A. Nakano, P. Vashishta, and S. Saini, Applied Physics Letters 77, 4332 (2000).

14. "Topology of amorphous gallium arsenide on intermediate length scales: a molecular dynamics study,” I. Ebbsjö, R. K. Kalia, A. Nakano, J. P. Rino, and P. Vashishta, Journal of Applied Physics 87, 7708 (2000).

15. "Role of atomic charge transfer on sintering of $\mathrm{TiO}_{2}$ nanoparticles: variable-charge molecular dynamics," S. Ogata, H. Iyetomi, K. Tsuruta, F. Shimojo, A. Nakano, R. K. Kalia, and P. Vashishta, Journal of Applied Physics 88, 6011 (2000).

16. "Large-scale atomistic modeling of nanoelectronic structures," A. Nakano, M. E. Bachlechner, P. Branicio, T. J. Campbell, I. Ebbsjö, R. K. Kalia, A. Madhukar, S. Ogata, A. Omeltchenko, J. P. Rino, F. Shimojo, P. Walsh, and P. Vashishta, IEEE Transactions on Electron Devices 47, 1804 (2000).

17. "A scalable molecular-dynamics-algorithm suite for materials simulations: design-space diagram on 1,024 Cray T3E processors," F. Shimojo, T. J. Campbell, R. K. Kalia, A. Nakano, P. Vashishta, S. Ogata, and K. Tsuruta, Future Generation Computer Systems 17, 279 (2000).

18. "Multiresolution algorithms for massively parallel molecular dynamics simulations of nanostructured materials," R. K. Kalia, T. J. Campbell, A. Chatterjee, A. Nakano, P. Vashishta, and S. Ogata, Computer Physics Communications 128, 245 (2000).

19. "Scalable I/O of large-scale molecular-dynamics simulations: a data-compression algorithm," A. Omeltchenko, T. J. Campbell, R. K. Kalia, X. Liu, A. Nakano, and P. Vashishta, Computer Physics Communications 131, 78 (2000).

20. "Multimillion atom simulations of nanostructured materials on parallel computers sintering and consolidation, fracture, and oxidation," P. Vashishta, M. E. Bachlechner, T. J. Campbell, R. K. Kalia, H. Kikuchi, S. Kodiyalam, A. Nakano, S. Ogata, F. Shimojo, and P. Walsh, Progress of Theoretical Physics Supplement 138, 175 (2000).

21. "Grain boundaries in gallium arsenide nanocrystals under pressure: a parallel moleculardynamics study," S. Kodiyalam, R. K. Kalia, H. Kikuchi, A. Nakano, F. Shimojo and P. Vashishta, Physical Review Letters 86, 55 (2001). 
22. "Coupling length scales for multiscale atomistic-continuum simulations: atomisticallyinduced stress distributions in $\mathrm{Si} / \mathrm{Si}_{3} \mathrm{~N}_{4}$ nanopixels," E. Lidorikis, M. E. Bachlechner, R. K. Kalia, A. Nakano, P. Vashishta, and G. Z. Voyiadjis, Physical Review Letters 87, 086104 (2001).

23. "Structual transformation, amorphization, and fracture in nanowires: a multi-million atom molecular dynamics study," P. Walsh, W. Li, R. K. Kalia, A. Nakano, P. Vashishta, and S. Saini, Applied Physics Letters 78, 3328 (2001).

24. "Million-atom molecular dynamics simulation of flat InAs overlayers with self-limiting thickness on GaAs nanomesas," X. Su, R. K. Kalia, A. Madhukar, A. Nakano, and P. Vashishta, Applied Physics Letters 78, 3717 (2001).

25. "Critical lateral size for domain formation in InAs/GaAs square nanomesas: a multimillion-atom molecular dynamics study," X. Su, R. K. Kalia, A. Madhukar, A. Nakano, and P. Vashishta, Applied Physics Letters 79, 4577 (2001).

26. "Multimillion atom simulation of materials on parallel computers-nanopixel, interfacial fracture, nanoindentation, and oxidation," P. Vashishta, M. E. Bachlechner, A. Nakano, T. J. Campbell, R. K. Kalia, S. Kodiyalam, S. Ogata, F. Shimojo, and P. Walsh, Applied Surface Science 182, 258 (2001).

27. "Multiscale simulation of nanosystems," A. Nakano, M. E. Bachlechner, R. K. Kalia, E. Lidorikis, P. Vashishta, G. Z. Voyiadjis, T. J. Campbell, S. Ogata, and F. Shimojo, IEEE Computing in Science and Engineering 3 (4), 56 (2001).

28. "Hybrid finite-element/molecular-dynamics/electronic-density-functional approach to materials simulations on parallel computers," S. Ogata, E. Lidorikis, F. Shimojo, A. Nakano, P. Vashishta, and R. K. Kalia, Computer Physics Communications 138, 143 (2001).

29. "Linear-scaling density-functional-theory calculations of electronic structure based on real-space grids: design, analysis, and scalability test of parallel algorithms," F. Shimojo, R. K. Kalia, A. Nakano, and P. Vashishta, Computer Physics Communications 140, 303 (2001).

30. "Pressure induced structural transformation in gallium arsenide: a molecular-dynamics study,” J. P. Rino, A. Chatterjee, I. Ebbsjö, R. K. Kalia, A. Nakano, F. Shimojo, and P. Vashishta, Physical Review B 65, 195206 (2002).

31. "Hybrid quantum mechanical/molecular dynamics simulation on parallel computers: density functional theory on real-space multigrids," S. Ogata, F. Shimojo, A. Nakano, P. Vashishta, and R. K. Kalia, Computer Physics Communications 149, 30 (2002).

32. "Scalable atomistic simulation algorithms for materials research," A. Nakano, R. K. Kalia, P. Vashishta, T. J. Campbell, S. Ogata, F. Shimojo, and S. Saini, Scientific Programming 10, 263 (2002). 
33. "Atomistic aspects of crack propagation in brittle materials: multimillion atom molecular dynamics simulations," C. L. Rountree, R. K. Kalia, E. Lidorikis, A. Nakano, L. Van Brutzel, and P. Vashishta, in Annual Review of Materials Research, Vol. 32, edited by D. R. Clarke and M. Rühle (Annual Reviews, Palo Alto, CA, 2002) p. 377.

§3 PARTICIPATION/PRESENTATIONS AT CONFERENCES, SEMINARS, ETC.

1. Multimillion Atom Simulations of Nanostructured Materials on ParallelComputers Sintering and Consolidation, Fracture, and Oxidation, International Conference on Computational Physics, Kanazawa, Japan, October 11-13, 1999.

2. Atomistic Simulations of Nanostructures: Multimillion Atom Molecular Dynamics Simulations on Parallel Computers, Foresight Conference on Molecular Nanotechnology, San Jose, CA, October 15-17, 1999.

3. Multimillion Atom Simulation of Nanostructured Materials on Parallel Computers Sintering and Consolidation and Fracture and Oxidation, The Materials Society (TMS), Nashville, TN, March 12-16, 2000.

4. Large-Scale Atomistic Simulations of Solid State Materials Modeling Many Millions of Atoms on Parallel Computers, American Physical Society, Minneapolis, March 22-24, 2000 .

5. Multimillion Atom Simulations of Materials on Parallel Computers - Past, Present and Future, XXIII Encontro Nacional de Física da Matéria Condensada, São Lourenço, Minas Gerais, Brasil, May 11-13, 2000.

6. Multi-Million Atom Simulation of Sintering and Consolidation on Parallel Computers, SIAM Conference on Mathematical Aspects of Materials Science, Philadelphia, PA, May 23, 2000.

7. Large-Scale Atomistic Simulations of Amorphous Polymers and Ceramic/Polymer Interfaces on Parallel Computers, American Crystallographic Society ”, Minneapolis, MN, July 22-27, 2000.

8. Multimillion Atom Simulation of Nanostructured Materials - Dynamic Fracture, Nanoindentation, and Oxidation, International Conference on Engineering \& Technological Sciences 2000 - Advanced Materials, Beijing, China, October 10-14, 2000.

9. Large Scale Molecular Dynamics Simulations of Materials on Parallel Computers, P. Vashishta, ACAT2000 Conference, Fermilab, IL, October 18-19, 2000. 
10. Large Scale Molecular Dynamics Simulations of Nanostructured Materials on Parallel Computers- Dynamic Fracture, Nanoindentation, and Oxidation, Joint CNRS (France)NSF (USA) Nanomaterials Workshop - Nanomaterials Toward Engineering Applications, Montreal, Canada, October 20-25, 2000.

11. Periodic Array of Stress Domains in Silicon/Silicon Nitride Nanopixels: A Multimillion Atom Molecular Dynamics Simulation, International Conference on Science and Technology of Nanostructured Materials, Puri, India, January 8-12, 2001.

12. Multimillion Atom Simulation of Nanosystems at the Forefront of Information Technology and Nanosciences, and An Integrated Dual Degree Program of Research and Education, National Science Foundation Distinguished Lecture", Arlington, VA, February 26, 2001.

13. Multimillion Atom Simulations of Mechanical Behavior of Nanostructured Materials, Interfaces, and Dynamics of Oxidation, The Society of Materials Science, Japan (JSMS), Osaka, Japan, May 21-26, 2001.

14. Multimillion Atom Simulations of Materials at the Forefront of Information Technology and Nanosciences, National Research Council, National Materials Advisory Board Meeting on Materials Research for Defense-After-Next, Woods Hole Center of the National Academy of Sciences, MA, June 27-28, 2001.

15. Multimillion Atom Simulations of Nanosystems on Parallel Computers, International Workshop on Condensed Matter Theories, Canberra, Australia, December 3-8, 2001.

16. Computational Materials Science on Teraflop-to-Petaflop Computers, DOD, DOE, NASA Joint Conference on Mission Critical Computing", Washington DC, February 4-6, 2002.

17. Multimillion Atom Simulations of Nanosystems on Parallel Computers, International Symposium on Computational Science \& Engineering”, Tokyo, Japan, March 5-6, 2002.

18. Multiscale FE/MD/QMD Method and Multimillion atom Simulations of Nanosystems and Interfaces on Parallel Computers, Symposium on Modeling and Numerical Simulation of Materials Behavior and Evolution, MRS Spring Meeting, CA, 2002.

19. Large-Scale Molecular Dynamics Simulations of the Oxidation of Metallic Nanoparticles, Gordon Conference on Energetic Materials, Tilton, NH, June 19, 2002.

20. Amorphous Materials in Various Incarnations, CECAM Workshop on Atomic Structure and Transport in Glassy Networks, Lyon, France, June 24-27, 2002.

21. Multimillion Atom Simulations of Nanosystems on Parallel Computers - Nanopixel, Nanoindentation and Oxidation, APS Conference on Computational Physics 2002, San Diego, Lyon, August 25-28, 2002.

22. Multimillion Atom Simulations of Nanosystems on Parallel Computers - Nanopixel, 
Nanoindentation and Oxidation of Aluminum Nanoparticles, CNER-MSI Nanosimulation Workshop" Center for Nanoenergetics Research, Minnesota Supercomputing Institute, Minneapolis, MN, August 26-27, 2002. 


\section{§4 INTERACTIONS WITH UNIVERSITIES, NATIONAL LABORATORIES AND INDUSTRIES}

Below we briefly describe the interactions we have had with researchers at universities, government laboratories and industries.

Argonne National Laboratory: We are collaborating with a number of scientists at Argonne National Laboratory in Illinois: Drs. C. Loong, D. Price, and M.-L. Saboungi, who are involved in neutron scattering measurements on ceramic and nanostructured materials at the Intense Pulsed Neutron Source Division; W. Sturhahn, T. S. Toellner, and E. E. Alp who are conducting experiments on oxides at the Advanced Photon Source; and T. Disz, W. Gropp, and R. Stevens who are designing parallel algorithms, message-passing, and immersive and interactive visualization tools.

Phillips Laboratory: We are collaborating with Drs. Joseph Lichtenhan and Stephen Rodgers on POSS (Polyhedral Oligomeric Silsesquioxane) based materials.

Nanophase Technologies: We are interacting with Dr. Richard Siegel at Nanophase Technologies. (This company synthesizes nanophase materials.)

Motorola: Recently we have started collaboration on sintering of multilayered ceramic films with Dr. D. Wilcox's experimental group at Ceramic Technology Center at Motorola.

\section{§5 TRAINING OF GRADUATE STUDENTS}

Our graduate students are enrolled in a unique, multidisciplinary program that allows them to obtain a Ph.D. in physics and an MS from the Department of Computer Science. The aim of this program is to provide students with broad-based training in high performance computing and communications (HPCC) and the physical sciences. In connection with this program, we have introduced a number of HPCC courses in the Physics and Computer Science Departments. The Department of Physics has two graduate courses in computational physics that are cross-listed with computer science courses. The first course deals with classical and quantum simulations on parallel architectures. The second course, designed for advanced graduate students, covers special topics such as multiscale phenomena, multigrid methods, wavelets, etc. In the Department of Computer Science, we have introduced three new HPCC courses. Soon two more courses will be added to the computer-science curriculum: a) Heterogeneous Computing; and b) Scientific Visualization. The courses we have introduced emphasize parallel computing and algorithm design for large-scale scientific applications. Students have access to a number of parallel machines to gain hands-on experience and to perform research on large-scale computational projects.

Our students have excellent opportunities to broaden their research experience beyond the traditional university based environment. They are involved in our collaborative efforts with computational and experimental scientists at government laboratories, industries, and other universities. These interactions have significantly enhanced the research capabilities of students. 
Through these contacts, students also have access to excellent parallel computing and visualization facilities at other institutions. One of the dual-degree students, Dr. Alok Chatterjee, supervised by the PIs of this program received a prestigious Enrico Fermi Fellowship from the US Department of Energy in 1999.

\section{§6 COMPUTATIONAL FACILITIES}

For the research program, our Concurrent Computing Laboratory for Materials Simulations (CCLMS) at Louisiana State University (LSU) has two parallel computing laboratories, one in the Department of Physics and Astronomy and the other in the Department of Computer Science. With \$3 million in infrastructure enhancement grants from the State of Louisiana, these labs have been equipped with several parallel machines including:

- Digital Alpha cluster - 64 Alpha processors connected via two Gigaswitches and a fast ethernet switch;

- PC cluster-165 PCs (800 MHz Pentium III) linked by a network fabric with gigabit and fast ethernet switches.

The CCLMS also has a number of parallel computers including a 64-cell Intel iWarp systolic architecture and an 8,192-node MasPar for teaching and experimenting with a diversity of architectures.

We have also established a virtual environment (VE) laboratory that features an interactive and immersive ImmersaDesk for visualization. The VE lab also has a multiprocessor SGI Onyx2/InfiniteReality2, an Octane/MXE, and an 8-processor Power Center graphics servers as well as a number of SGI graphics workstations. Via high-speed networks, the ImmersaDesk is fully integrated with the existing parallel machines at the CCLMS and with massively parallel computers at national computer centers. 